\title{
Total Synthesis of (+)-Cocaine via Desymmetrization of a Meso-Dialdehyde
}

\author{
Douglas M. Mans and William H. Pearson* ${ }^{\dagger}$ \\ Department of Chemistry, University of Michigan, Ann Arbor, MI 48109-1055, USA \\ Email: wpearson@berryassoc.com \\ $†$ Current Address: Berry and Associates, Inc., 2434 Bishop Circle East, Dexter, MI, \\ 48130 USA.
}

\section{Supporting Information}

\author{
Table of Contents \\ Experimental procedures for compounds (+)-1, and 6-15. \\ pages: S2-S14 \\ ${ }^{1} \mathrm{H}$ NMR spectra for compounds (+)-1, and 6-15. \\ pages: S15-S26
}




\section{EXPERIMENTAL}

General Methods. All reactions were performed under an atmosphere of dry nitrogen or argon in flame-dried glassware equipped with tightly fitting rubber septa. All syringes and needles were oven-dried and cooled in a dessicator prior to use. Reactions at $0{ }^{\circ} \mathrm{C}$ were carried out in an ice/water bath. Reactions at $-78{ }^{\circ} \mathrm{C}$ were carried out in a dry ice/acetone bath. Solvents were freshly prepared before use. Benzene, toluene, methylene chloride $\left(\mathrm{CH}_{2} \mathrm{Cl}_{2}\right)$, pyridine, triethylamine and diisopropylamine were distilled from calcium hydride. Tetrahydrofuran (THF) and diethyl ether were distilled from sodium/benzophenone ketyl. Dimethylformamide (DMF) was distilled from barium oxide under reduced pressure. $n$-Butyllithium was titrated with diphenylacetic acid prior to use.

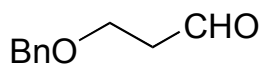

3-Benzyloxypropionaldehyde (6): Dimethyl sulfoxide (3.90 mL, $4.29 \mathrm{~g}, 55 \mathrm{mmol})$ was added in a dropwise fashion to a solution of oxalyl chloride $(4.40 \mathrm{~mL}, 6.40 \mathrm{~g}, 50 \mathrm{mmol})$ in methylene chloride $(240 \mathrm{~mL})$ cooled to $-78{ }^{\circ} \mathrm{C}$. The solution was stirred for $15 \mathrm{~min}$ followed by dropwise addition of a solution of 3-benzyloxy-1-propanol $(4.00 \mathrm{~mL}, 4.20 \mathrm{~g}$, $25 \mathrm{mmol})$ in methylene chloride $(10 \mathrm{~mL})$. After addition was complete the mixture was stirred an additional $15 \mathrm{~min}$. Triethylamine $(21.0 \mathrm{~mL}, 15.2 \mathrm{~g}, 150 \mathrm{mmol})$ was then added in a dropwise fashion and the mixture allowed to stir for a further $15 \mathrm{~min}$. Following this the cold bath was removed and the mixture allowed to warm to room temperature. The mixture was diluted with diethyl ether and washed with water $(3 \mathrm{x})$, dried $\left(\mathrm{MgSO}_{4}\right)$, filtered and concentrated to an oil. Chromatography (20\% diethyl ether/ pentanes) yielded $4.06 \mathrm{~g}$ (99\%) of 3-benzyloxypropionaldehyde 6 as a oil. ${ }^{1} \mathrm{H}$ NMR matched that 
reported in the literature: Kozikowski, A. P.; Stein, P. D. J. Org. Chem. 1984, 49(13), 2301-2309. ${ }^{1} \mathrm{H}$ NMR (500 MHz, $\left.\mathrm{CDCl}_{3}\right) \delta 9.80(\mathrm{t}, 1 \mathrm{H}, J=2.0 \mathrm{~Hz}), 7.28(\mathrm{~s}, 5 \mathrm{H}), 4.46$ (s, $2 \mathrm{H}), 3.74(\mathrm{t}, 2 \mathrm{H}, J=6.0 \mathrm{~Hz}), 2.60(\mathrm{dt}, 2 \mathrm{H}, J=6.0,2.0 \mathrm{~Hz})$.

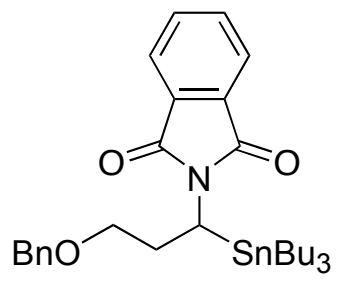

$N$-[1-(Tri- $n$-butylstannyl)-3-benzyloxypropyl]phthalimide (7): $n$-Butyllithium (19.4 $\mathrm{mL}$ of a $2.5 \mathrm{M}$ solution in hexanes, $48.4 \mathrm{mmol}$ ) was added in a dropwise fashion to a solution of hexabutylditin $(24.5 \mathrm{~mL}, 28.1 \mathrm{~g}, 48.4 \mathrm{mmol}))$ in $\mathrm{THF}(190 \mathrm{~mL})$ at $-78{ }^{\circ} \mathrm{C}$. The solution was warmed to $0{ }^{\circ} \mathrm{C}$ and allowed to stir for $30 \mathrm{~min}$. The mixture was then cooled to $-78{ }^{\circ} \mathrm{C}$ and a solution of 3-Benzyloxypropionaldehyde 6 (6.61 g, $\left.40.3 \mathrm{mmol}\right)$ in THF $(10 \mathrm{~mL})$ was then added in a dropwise fashion and the mixture was stirred for $1 \mathrm{~h}$. Saturated aqueous ammonium chloride then added to the solution and allowed to warm to room temperature. The layers were separated and the aqueous layer was extracted with diethyl ether (3x). The organic extracts were combined, dried $\left(\mathrm{Na}_{2} \mathrm{SO}_{4}\right)$, filtered and concentrated. Chromatography (5-10\% ethyl acetate/hexanes gradient) gave $8.89 \mathrm{~g}$ $(50 \%)$ of the intermediate stannyl alcohol as a clear oil. Data for the stannyl alcohol: ${ }^{1} \mathrm{H}$ NMR (400 MHz, $\left.\mathrm{CDCl}_{3}\right) \delta 7.33(\mathrm{~s}, 5 \mathrm{H}), 4.52(\mathrm{~s}, 2 \mathrm{H}), 4.28(\mathrm{ddd}, 1 \mathrm{H}, J=9.6,7.2,3.2$ Hz), 3.76-3.65 (m, 2 H), 2.19-2.10 (m, 1 H), 2.04-1.97 (m, 1 H), 1.54-1.46 (m, $6 \mathrm{H}), 1.30$ (sextet, $6 \mathrm{H}, J=7.2 \mathrm{~Hz}$ ), $0.89(\mathrm{t}, 15 \mathrm{H}, J=7.2 \mathrm{~Hz}$ ).

The stannyl alcohol (7.29 g, $16 \mathrm{mmol})$ was then dissolved in THF (75 mL) along with triphenylphosphine $(4.62 \mathrm{~g}, 17.6 \mathrm{mmol})$ and phthalimide $(2.60 \mathrm{~g}, 17.6 \mathrm{mmol})$. The 
mixture was cooled to $0{ }^{\circ} \mathrm{C}$ and diethylazodicarboxylate $(2.80 \mathrm{~mL}, 3.10 \mathrm{~g}, 17.6 \mathrm{mmol})$ in THF ( $5 \mathrm{~mL}$ ) was then added dropwise. The bright yellow solution was allowed to slowly warm to room temperature and stir overnight. The solvent was removed in vacuo. The residue was then chromatographed (0-5\% ethyl acetate/hexanes) to give $9.18 \mathrm{~g}(98 \%)$ of 7 as a yellow oil. Data for 7: $\mathrm{R}_{f}=0.43$ (10\% ethyl acetate/hexanes); ${ }^{1} \mathrm{H}$ NMR (500 $\left.\mathrm{MHz}, \mathrm{CDCl}_{3}\right) \delta 7.76(\mathrm{dd}, 2 \mathrm{H}, J=5.0,3.0 \mathrm{~Hz}), 7.66(\mathrm{dd}, 2 \mathrm{H}, J=5.0,3.0 \mathrm{~Hz}), 7.24-7.19$ (m, $5 \mathrm{H}), 4.38(\mathrm{dd}, 2 \mathrm{H}, J=19.0,11.8 \mathrm{~Hz}), 4.08(\mathrm{dd}, 1 \mathrm{H}, J=10.5,5.5 \mathrm{~Hz}), 3.45(\mathrm{t}, 2 \mathrm{H}$, $J=6.5 \mathrm{~Hz}), 2.28(\mathrm{ddt}, 1 \mathrm{H}, J=17.5,10.5,6.0 \mathrm{~Hz}), 2.01(\mathrm{ddt}, 1 \mathrm{H}, J=14.6,6.5,5.0 \mathrm{~Hz})$, 1.49-1.43 (m, $6 \mathrm{H}), 1.26(\mathrm{sex}, 6 \mathrm{H}, J=7.5 \mathrm{~Hz}), 0.92(\mathrm{dd}, 6 \mathrm{H}, J=8.5,8.0 \mathrm{~Hz}), 0.83(\mathrm{t}, 9$ $\mathrm{H}, J=7.5 \mathrm{~Hz}) ;{ }^{13} \mathrm{C}$ NMR $\left(100 \mathrm{MHz}, \mathrm{CDCl}_{3}\right) \delta 169.4,138.5,133.8,132.3,128.4,127.8$, 123.0, 73.3, 69.8, 34.8, 33.0, 29.1, $27.6\left(J_{\mathrm{Sn}^{17}}{ }_{-\mathrm{C}}{ }^{13}=29.6 \mathrm{~Hz}\right), 13.8,10.6\left(J_{\mathrm{Sn}}{ }^{117}{ }_{-\mathrm{C}}{ }^{13}=163.1\right.$ Hz); IR (neat) 1770 (m), 1701 (s), 1611 (w), 1465 (m), 1454 (m), 1387 (s), 1356 (s) cm' MS (EI, $70 \mathrm{eV}) \mathrm{m} / \mathrm{z}$ (rel int) $528.2\left(100,[\mathrm{~m}-57]^{+}\right), 265.9$ (15), 179.0 (23), 91.1 (89); HRMS (ES, pos. ion) calcd for $\mathrm{C}_{30} \mathrm{H}_{43} \mathrm{NO}_{3} \mathrm{SnNa}[\mathrm{M}+\mathrm{Na}]^{+}$608.2163; found 608.2192.

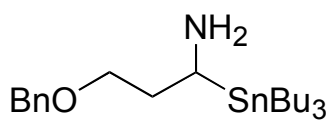

3-Benzyloxy-1-(tri-n-butylstannyl)propylamine (8): Hydrazine monohydrate (10.5 $\mathrm{mL}, 10.8 \mathrm{~g}, 215 \mathrm{mmol}$ ) was added in dropwise fashion to a solution of phthalimide 7 (2.5 $\mathrm{g}, 4.3 \mathrm{mmol})$ in ethanol $(11.0 \mathrm{~mL})$ at reflux. After $16 \mathrm{~h}$ the solution was cooled to room temperature and diluted with diethyl ether. The mixture was washed with water (5x) and brine (1x), dried $\left(\mathrm{Na}_{2} \mathrm{SO}_{4}\right)$ and concentrated to yield $1.88 \mathrm{~g}(96 \%)$ of $\mathbf{8}$ as a clear oil. Used directly with no further purification. Data for 8: ${ }^{1} \mathrm{H} \mathrm{NMR}\left(500 \mathrm{MHz}, \mathrm{CDCl}_{3}\right) \delta$ 
7.34-7.33 (m, $5 \mathrm{H}), 4.52(\mathrm{~d}, 1 \mathrm{H}, J=12.0 \mathrm{~Hz}), 4.48(\mathrm{~d}, 1 \mathrm{H}, J=12.0 \mathrm{~Hz}), 3.60-3.54(\mathrm{~m}, 2$ H), $3.00(\mathrm{dd}, 1 \mathrm{H}, J=8.5,5.0 \mathrm{~Hz}), 2.02-1.89(\mathrm{~m}, 2 \mathrm{H}), 1.54-1.41(\mathrm{~m}, 6 \mathrm{H}), 1.30(\mathrm{sex}, 6$ $\mathrm{H}, J=7.5 \mathrm{~Hz}), 0.89(\mathrm{t}, 9 \mathrm{H}, J=7.5 \mathrm{~Hz}), 0.88-0.85(\mathrm{~m}, 6 \mathrm{H})$.

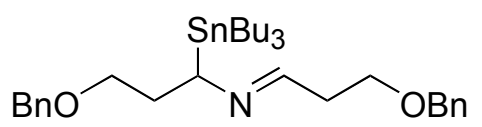

\section{(3-Benzyloxypropylidene)-[3-benzyloxy-1-(tri-n-butylstannyl)propyl]amine (9):}

Stannyl amine 8 (1.80 g, $4.0 \mathrm{mmol})$ was dissolved in diethyl ether $(35 \mathrm{~mL})$ and $\sim 0.5 \mathrm{~g}$ of $4 \AA ̊$ molecular sieves added. A solution of 3-benzyloxypropionaldehyde $(0.651 \mathrm{~g}, 4.0$ mmol) in diethyl ether $(5 \mathrm{~mL})$ was added dropwise. After $2 \mathrm{~h}$ the solution was filtered through celite and concentrated to yield $2.3 \mathrm{~g}(95 \%)$ of 9 as a clear oil. Data for 9 : ${ }^{1} \mathrm{H}$ NMR (500 MHz, $\left.\mathrm{CDCl}_{3}\right) \delta 7.57(\mathrm{t}, 1 \mathrm{H}, J=4.5 \mathrm{~Hz}), 7.35-7.27(\mathrm{~m}, 10 \mathrm{H}), 4.51(\mathrm{t}, 2 \mathrm{H}, J$ $=14.5 \mathrm{~Hz}), 4.48(\mathrm{~d}, 1 \mathrm{H}, J=12.0 \mathrm{~Hz}), 4.41(\mathrm{~d}, 1 \mathrm{H}, J=12.0 \mathrm{~Hz}), 3.70(\mathrm{dd}, 1 \mathrm{H}, J=11.0$, $3.5 \mathrm{~Hz}$ ), 3.64 (td, $2 \mathrm{H}, J=6.5,1.5 \mathrm{~Hz}), 3.46$ (ddd, $1 \mathrm{H}, J=9.0,7.3,4.5 \mathrm{~Hz}), 3.38-3.33$ (m, $1 \mathrm{H}), 2.54-2.47$ (m $2 \mathrm{H}), 2.30$ (dddd, $1 \mathrm{H}, J=15.9,9.2,6.8,5.0 \mathrm{~Hz}$ ), 2.06 (dtd, $1 \mathrm{H}, J$ $=14.0,7.5,3.5 \mathrm{~Hz}), 1.49-1.43(\mathrm{~m}, 6 \mathrm{H}), 1.28(\mathrm{sex}, 6 \mathrm{H}, J=7.5 \mathrm{~Hz}), 0.88(\mathrm{t}, 9 \mathrm{H}, J=7.0$ $\mathrm{Hz}), 0.87-0.84(\mathrm{~m}, 6 \mathrm{H}) ;{ }^{13} \mathrm{C} \mathrm{NMR}\left(100 \mathrm{MHz}, \mathrm{CDCl}_{3}\right) \delta 155.4,138.8,138.5,128.6$, $128.5,127.9,127.8,127.7,127.6,73.1,69.6,68.1,59.1,36.2,34.4,29.3,27.7,13.9,9.0$; IR (neat) 1645 (w), 1453 (m), 1361 (m), 1101 (s, br) cm ${ }^{-1}$; MS (EI, $\left.70 \mathrm{eV}\right) \mathrm{m} / z$ (rel int) $544.1\left(5,[\mathrm{M}-57]^{+}\right), 436.1$ (12), 235.0 (16), 178.9 (21), 91.0 (100); HRMS (ES) calcd for $\mathrm{C}_{32} \mathrm{H}_{51} \mathrm{NO}_{2} \mathrm{SnNa}[\mathrm{M}+\mathrm{Na}]^{+}$624.2839; found 624.2860. 

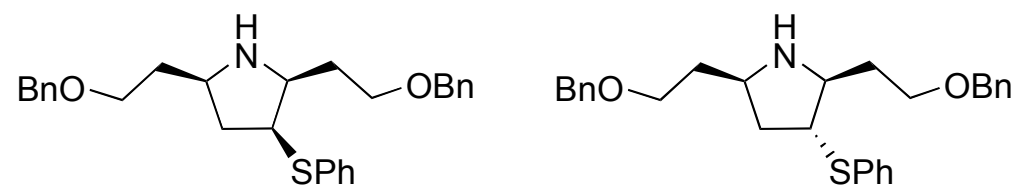

$\left(2 R^{*}, 3 R^{*}, 5 S^{*}\right)-2,5-\operatorname{Bis}\left(2-\right.$ benzyloxyethyl)-3-phenylsulfanyl pyrrolidine and ( $2 R^{*}$, $\left.3 S^{*}, 5 S^{*}\right)-2,5$-Bis(2-benzyloxyethyl)-3-phenylsulfanyl pyrrolidine (10): A solution of $9(0.97 \mathrm{~g}, 1.6 \mathrm{mmol})$ and phenyl vinyl sulfide $(0.42 \mathrm{~mL}, 0.43 \mathrm{~g}, 3.2 \mathrm{mmol})$ in THF (2.0 $\mathrm{mL})$ was added in a dropwise fashion to a solution of $n$-butyllithium $(0.97 \mathrm{~mL}$ of a $2.5 \mathrm{M}$ solution in hexanes, $2.4 \mathrm{mmol})$ in THF $(14 \mathrm{~mL})$ at $-78{ }^{\circ} \mathrm{C}$. After $30 \mathrm{~min}$., the mixture was quenched with water and warmed to room temperature. The solution was washed with water $(2 \mathrm{x})$ and brine $(1 \mathrm{x})$. The organic layer was then dried $\left(\mathrm{Na}_{2} \mathrm{SO}_{4}\right)$ and concentrated to a oil. Chromatography (100\% ethyl acetate) provided $0.729 \mathrm{~g}(97 \%)$ of 10 as a clear oil. Data for 10: $\mathrm{R}_{f}=0.14$ (50\% ethyl acetate/hexanes); ${ }^{1} \mathrm{H}$ NMR $(500 \mathrm{MHz}$, $\left.\mathrm{CDCl}_{3}\right) \delta$ 7.34-7.25 (m, $\left.15 \mathrm{H}\right), 4.48-4.45(\mathrm{~m}, 4 \mathrm{H}), 3.74(\mathrm{dt}, 1 \mathrm{H}, J=8.0,5.0 \mathrm{~Hz}), 3.58-$ $3.50(\mathrm{~m}, 4.5 \mathrm{H}), 3.40(\mathrm{dt}, 0.5 \mathrm{H}, J=8.5,5.5 \mathrm{~Hz}), 3.33-3.27$ (m, $1 \mathrm{H}), 3.22$ (pent, $0.5 \mathrm{H}, J$ $=7.5 \mathrm{~Hz}), 3.07(\mathrm{ddd}, 0.5 \mathrm{H}, J=8.5,7.0,4.5 \mathrm{~Hz}), 2.42(\mathrm{dt}, 1 \mathrm{H}, J=13.5,7.5 \mathrm{~Hz}), 2.03-$ $1.64(\mathrm{~m}, 6 \mathrm{H}), 1.56$ (ddd, $1 \mathrm{H}, J=13.3,7.5,5.0 \mathrm{~Hz}), 1.48-1.45$ (m, $0.5 \mathrm{H}), 1.31$ (pent, 0.5 H, $J=7.0 \mathrm{~Hz}$ ); IR (neat) 3346 (w), 1583 (w), 1479 (m), 1452 (m), 1437 (m), 1362 (m), 1097 (s, br) $\mathrm{cm}^{-1} ; \quad$ MS (EI, $\left.70 \mathrm{eV}\right) \mathrm{m} / z$ (rel int) 356.2 (18, [M-91] $\left.]^{+}\right), 312.2$ (43), 91.0 (100); HRMS (ES) calcd for $\mathrm{C}_{28} \mathrm{H}_{34} \mathrm{NO}_{2} \mathrm{~S}[\mathrm{M}+\mathrm{H}]^{+}$448.2310; found 448.2317. 


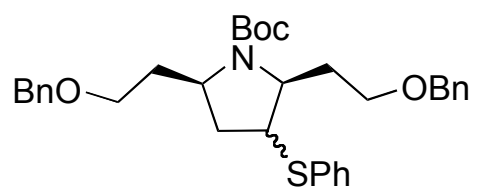

$\left(2 R^{*}, 3 R^{*}, 5 S^{*}\right)$-2,5-Bis(2-benzyloxyethyl)-3-phenylsulfanyl pyrrolidine-1-carboxyilc acid tert-butyl ester and $\left(2 R^{*}, 3 S^{*}, 5 S^{*}\right)$-2,5-Bis(2-benzyloxyethyl)-3-phenylsulfanyl pyrrolidine-1-carboxylic acid tert-butyl ester (11): A solution of di-tert-butyl dicarbonate $(284 \mathrm{mg}, 1.3 \mathrm{mmol})$ in methylene chloride $(1.3 \mathrm{~mL})$ was added to a vigorously stirred solution of pyrrolidine $\mathbf{1 0}(118 \mathrm{mg}, 0.26 \mathrm{mmol})$ and sodium bicarbonate $(439 \mathrm{mg}, 5.2 \mathrm{mmol})$ in water $(1.3 \mathrm{~mL})$. After $7 \mathrm{~h}$ the solution was diluted with methylene chloride, washed with water (3x) and brine (1x), dried $\left(\mathrm{MgSO}_{4}\right)$ and concentrated to an oil. Chromatography of the residue (5\% ethyl acetate/hexanes) yielded $108 \mathrm{mg}(75 \%)$ of $\mathbf{1 1}$ as a viscous clear colorless oil. Data for $\mathbf{1 1}: \mathbf{R}_{f}=0.23(10 \%$ ethyl acetate/hexanes); ${ }^{1} \mathrm{H}$ NMR $\left(500 \mathrm{MHz}, \mathrm{CDCl}_{3}\right) \delta$ 7.36-7.24 (m, $\left.15 \mathrm{H}\right), 4.51-4.43(\mathrm{~m}$, 4H), 3.86 (br s, $1 \mathrm{H}), 3.70$ (br s, $0.5 \mathrm{H}), 3.60$ (sex, $1 \mathrm{H}, J=6.5 \mathrm{~Hz}$ ), 3.49 (br s, $3.5 \mathrm{H}$ ), $2.50(\mathrm{dt}, 0.5 \mathrm{H}, J=12.5,7.5 \mathrm{~Hz}), 2.40-2.11(\mathrm{~m}, 3.5 \mathrm{H}), 1.92(\mathrm{br} \mathrm{s}, 0.5 \mathrm{H}), 1.84-1.77$ (m, 1 $\mathrm{H}), 1.69-1.58(\mathrm{~m}, 1.5 \mathrm{H}), 1.41(\mathrm{br} \mathrm{s}, 9 \mathrm{H}) ;{ }^{13} \mathrm{C} \mathrm{NMR}\left(100 \mathrm{MHz}, \mathrm{CDCl}_{3}\right) \delta 155.3,135.5$, 135.0, 132.2, 131.7, 130.6, 129.3, 129.2, 128.7, 128.5, 128.0, 127.9, 127.7, 127.3, 126.9, $82.2,79.7,73.3,73.2,73.1,68.6,68.1,66.1,55.7,44.1,37.4,31.9,28.7,28.6,28.2,15.5$; IR (neat) 1689 (s), 1386 (s), 1364 (s), 1166 (m, br), 1099 (s, br) cm ${ }^{-1}$; MS (EI, 70 eV) m/z (rel int) 446.2 (14), 356.2 (18), 312.1 (32), 91.1 (100); HRMS (ES) calcd for $\mathrm{C}_{33} \mathrm{H}_{41} \mathrm{NO}_{4} \mathrm{SNa}[\mathrm{M}+\mathrm{Na}]^{+}$570.2654; found 570.2655. 


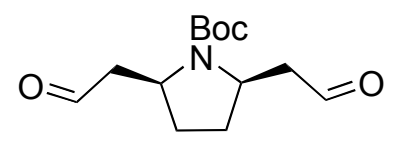

$\left(2 R^{*}, 5 S^{*}\right)-2,5$-Bis(oxoethyl)pyrrolidine-1- carboxylic acid tert-butyl ester (12):

Under argon pyrrolidine 11 (737 mg, $1.35 \mathrm{mmol})$ was dissolved in THF $(4.0 \mathrm{~mL})$ and cooled to $-78{ }^{\circ} \mathrm{C}$. Ammonia $(\sim 4.0 \mathrm{~mL})$ condensed into the solution with a cold finger. Lithium metal added portion-wise until a deep blue color formed. After stirring an additional 15 min the solution was quenched with solid ammonium chloride and allowed to warm to room temperature. The mixture was diluted with ether, dried $\left(\mathrm{Na}_{2} \mathrm{SO}_{4}\right)$, filtered and concentrated. Chromatography (50\% ethyl acetate/hexanes) yielded $346 \mathrm{mg}$ $(98 \%)$ of $\left(2 R^{*}, 5 S^{*}\right)$-Bis(2-hydroxyethyl)pyrrolidine carboxylic acid tert-butyl ester as a clear colorless oil. Data for $\left(2 R^{*}, 5 S^{*}\right)$-Bis(2-hydroxyethyl)pyrrolidine carboxylic acid tert-butyl ester: $\mathrm{R}_{f}=0.14$ (50\% ethyl acetate/hexanes); ${ }^{1} \mathrm{H}$ NMR $\left(500 \mathrm{MHz}, \mathrm{CDCl}_{3}\right) \delta$ 4.27 (br s, $1 \mathrm{H}), 4.18$ (br s, $1 \mathrm{H}), 3.95$ (br s, $1 \mathrm{H}), 3.64$ (br s, $4 \mathrm{H}), 2.10-2.07$ (m, $2 \mathrm{H}$ ), 1.95 (br s, $1 \mathrm{H}), 1.77-1.62$ (m, $6 \mathrm{H}), 1.49(\mathrm{~s}, 9 \mathrm{H}) ;{ }^{13} \mathrm{C}$ NMR (100 MHz, $\left.\mathrm{CDCl}_{3}\right) \delta 156.8$, 80.9, 60.2, 59.5, 39.5, 31.0, 28.6; IR (neat) 3407 (m, br), 1658 (s), 1398 (s), 1165 (m), 1110 (m), 1046 (m) cm $\mathrm{cm}^{-1}$, MS (EI, $70 \mathrm{eV}$ ), m/z (rel int) 214.2 (7), 158.1 (16), 114.1 (100), 57.1 (72); HRMS (ES) calcd for $\mathrm{C}_{13} \mathrm{H}_{25} \mathrm{NO}_{4} \mathrm{Na}[\mathrm{M}+\mathrm{Na}]^{+} 282.1681$; found 282.1681. Tetra- $n$-propylammonium perruthenate $(2.5 \mathrm{mg}, 0.007 \mathrm{mmol})$ was added in one portion to a solution of $\left(2 R^{*}, 5 S^{*}\right)$-Bis(2-hydroxyethyl)pyrrolidine carboxylic acid tert-butyl ester (17.8 $\mathrm{mg}, 0.07 \mathrm{mmol})$ and 4-methylmorpholine $N$-oxide $(18.0 \mathrm{mg}, 0.15 \mathrm{mmol})$ in methylene chloride $(0.5 \mathrm{~mL})$. After $1 \mathrm{~h}$ the solution was filtered through a pad of silica using ethyl acetate as eluent. The organic phases were dried $\left(\mathrm{Na}_{2} \mathrm{SO}_{4}\right)$ and concentrated to give $11 \mathrm{mg}(62 \%)$ of $\mathbf{1 2}$ as a clear colorless oil. Data for $\mathbf{1 2}: \mathrm{R}_{f}=0.51$ (50\% ethyl 
acetate/hexanes); ${ }^{1} \mathrm{H}$ NMR (500 MHz, $\left.\mathrm{CDCl}_{3}\right) \delta 9.76$ (s, $\left.2 \mathrm{H}\right), 4.33-4.24(\mathrm{~m}, 2 \mathrm{H}), 3.02-$ 2.78 (m, 2 H), 2.52 (ddd, 2 H, $J=16.5,7.5,1.5 \mathrm{~Hz}), 2.21-2.11$ (m, $2 \mathrm{H}), 1.69-1.63$ (m, 2 $\mathrm{H}), 1.43(\mathrm{~s}, 9 \mathrm{H}) ;{ }^{13} \mathrm{C}$ NMR $\left(100 \mathrm{MHz}, \mathrm{CDCl}_{3}\right) \delta 200.7,154.5,80.9,60.6,53.8,30.8$, 28.6; IR (neat) 1721 (s), 1686 (s), 1391 (s), 1366 (s) cm ${ }^{-1}$; MS (CI) m/z (rel int) 256.2 (3, $\left.[\mathrm{M}+\mathrm{H}]^{+}\right), 200.2(100), 112.1(18)$; HRMS (CI) calcd for $\mathrm{C}_{13} \mathrm{H}_{22} \mathrm{NO}_{4}[\mathrm{M}+\mathrm{H}]^{+}$256.1549; found 256.1553 .

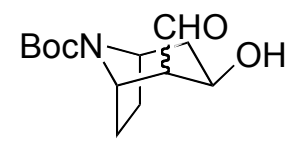

$(1 S, 2 S, 3 R, 5 R)$-2-Formyl-3-hydroxy-8-azabicyclo[3.2.1]octane-8-carboxylic acid tertbutyl ester and $(1 S, 2 R, 3 R, 5 R)$-2-Formyl-3-hydroxy-8-azabicyclo[3.2.1]octane-8carboxylic acid tert-butyl ester (13ax/eq): A solution of L-proline (24.5 mg, 0.21 mmol) and dialdehyde $12(216.5 \mathrm{mg}, 0.85 \mathrm{mmol})$ in toluene $(8.5 \mathrm{~mL})$ was stirred at room temperature for $24 \mathrm{~h}$. The mixture was then diluted with diethyl ether and washed with water $(2 \mathrm{x})$ and brine $(1 \mathrm{x})$. The organic layer was then dried $\left(\mathrm{Na}_{2} \mathrm{SO}_{4}\right)$, filtered and concentrated to give $216.5 \mathrm{mg}$ of the aldol product $(\mathrm{C}(2)$ epimers13ax and 13eq; $1: 1)$ as a clear colorless oil. The aldol products $13 \mathbf{a x} / \mathbf{e q}$ were carried on directly to the oxidation/esterification step without further purification due to their instability. Data for 13ax/eq: $\mathrm{R}_{f}=0.29\left(50 \%\right.$ ethyl acetate/hexanes); ${ }^{1} \mathrm{H}$ NMR $\left(500 \mathrm{MHz}, \mathrm{CDCl}_{3}\right) \delta 9.82-9.75$ (m, $1 \mathrm{H}), 4.37-4.24(\mathrm{~m}, 2 \mathrm{H}), 4.07$ (br s, $0.5 \mathrm{H}), 3.93$ (br s, 0.5 H), 2.00-1.95 (m, $3 \mathrm{H})$, 1.68-1.63 (m, $4 \mathrm{H}), 1.48$ (s, $4.5 \mathrm{H}), 1.43(\mathrm{~s}, 4.5 \mathrm{H}) ;{ }^{13} \mathrm{C} \mathrm{NMR}\left(100 \mathrm{MHz}, \mathrm{CDCl}_{3}\right) \delta 191.0$, $154.6,80.4,63.5,53.7,52.6,52.3,49.1,48.1,38.8,38.6,30.5,29.9,29.4,28.6,28.5$, 28.4; IR (neat) 3422 (m, br), 1720 (s), 1689 (s), 1392 (s, br) cm ${ }^{-1}$; MS (EI, $70 \mathrm{eV}$ ) m/z (rel 
int) $227.2\left(2,[\mathrm{M}-\mathrm{CHO}]^{+}\right), 153.1(13), 83.1$ (31), 57.1 (100); HRMS (ES) calcd for $\mathrm{C}_{14} \mathrm{H}_{25} \mathrm{NO}_{5} \mathrm{Na}[\mathrm{M}+\mathrm{Na}+\mathrm{MeOH}]^{+}$310.1630; found 310.1628.

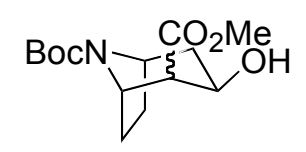

$(1 S, 2 S, 3 R, 5 R)$-3-Hydroxy-8-azabicyclo[3.2.1]octane-2,8-dicarboxylic acid-8-tertbutyl ester-2-methyl ester and $(1 S, 2 R, 3 R, 5 R)-3$-Hydroxy-8-azabicyclo[3.2.1]octane2,8-dicarboxylic acid-8-tert-butyl ester-2-methyl ester (14ax/eq): Aldol 13ax/eq (216 $\mathrm{mg}, 0.85 \mathrm{mmol})$ and 2-methyl-2-butene $(2.98 \mathrm{~g}, 4.50 \mathrm{~mL}, 42.5 \mathrm{mmol})$ were dissolved in 2-methyl-2-propanol (7.55 mL). A solution of sodium chlorite $(692 \mathrm{mg}, 7.65 \mathrm{mmol})$ and monobasic sodium phosphate monohydrate $(821 \mathrm{mg}, 5.95 \mathrm{mmol})$ in water $(0.95 \mathrm{~mL})$ was added dropwise to the mixture at room temperature. After stirring for 30 min the solvent was removed in vacuo. The residue was taken up in water and extracted with ethyl acetate $(3 \mathrm{x})$. The extracts were dried $\left(\mathrm{MgSO}_{4}\right)$, filtered and concentrated to give $234 \mathrm{mg}$ of crude carboxylic acid. The crude acid was taken up in a diethyl ether $(13 \mathrm{~mL})$ and cooled to $0{ }^{\circ} \mathrm{C}$. A solution of diazomethane was prepared by adding $N$-methyl- $N$ nitrosourea $(1.30 \mathrm{~g}, 12.7 \mathrm{mmol})$ portionwise to a $40 \mathrm{wt} / \mathrm{vol} \%$ aqueous $\mathrm{KOH}(2.4 \mathrm{~mL})$ and diethyl ether $(4 \mathrm{~mL})$ solution cooled to $0{ }^{\circ} \mathrm{C}$. The mixture was stirred until no more of the urea remained. The solution was then allowed to stand for 10 min afterwhich the bright yellow ether layer was decanted off and added dropwise to the chilled solution of the carboxylic acid. The mixture was stirred at $0{ }^{\circ} \mathrm{C}$ for $5 \mathrm{~min}$, warmed to room temperature and stirred an additional $10 \mathrm{~min}$. Glacial acetic acid was then added dropwise until the yellow color dissipated. The mixture was diluted with ether and washed with saturated aq. $\mathrm{NaHCO}_{3}(3 \mathrm{x})$. The aqueous phase was then back-extracted 
with ether $(2 \mathrm{x})$. The organic phases were dried $\left(\mathrm{MgSO}_{4}\right)$ and concentrated to give 185 mg (76\% starting from dialdehyde $\mathbf{1 2}$ ) of $\mathbf{1 4 a x} / \mathbf{e q}$ as a clear oil. ${ }^{1} \mathrm{H}$ NMR showed the product was a mixture (1:1) of $\mathrm{C}(2)$ epimers Data for $\mathbf{1 4 a x} / \mathbf{e q}: \mathrm{R}_{f}=0.13$ (50\% ethyl acetate/hexanes); ${ }^{1} \mathrm{H} \mathrm{NMR}\left(500 \mathrm{MHz}, \mathrm{CDCl}_{3}\right) \delta 4.31-4.21(\mathrm{~m}, 2 \mathrm{H}), 4.19-4.00(\mathrm{~m}, 3 \mathrm{H})$, 3.75 (br s, $1.5 \mathrm{H}), 3.72$ ( br s, $1.5 \mathrm{H}), 3.66$ (s, $3 \mathrm{H}), 3.05-2.78$ (m, $2 \mathrm{H}), 2.90(\mathrm{dd}, 1 \mathrm{H}, J=$ 5.5, $2.0 \mathrm{~Hz}), 2.35-2.29$ (m, $2 \mathrm{H}), 2.10-1.87$ (m, $6 \mathrm{H}), 1.74-1.58(\mathrm{~m}, 6 \mathrm{H}), 1.47$ (s, $4.5 \mathrm{H})$, $1.46(\mathrm{~s}, 4.5 \mathrm{H}), 1.45$ (s, $4.5 \mathrm{H}), 1.43(\mathrm{~s}, 4.5 \mathrm{H}) ;{ }^{13} \mathrm{C} \mathrm{NMR}\left(100 \mathrm{MHz}, \mathrm{CDCl}_{3}\right) \delta 171.7$, $171.3,154.5,154.2,79.9,79.8,79.5,64.6,64.1,55.9,55.3,55.25,55.22,53.4,52.0,51.8$, 51.5, 38.3, 28.4, 28.3, 21.0, 20.7, 20.5, 19.9; IR (neat) 3420 (m, br), 1713 (s), 1693 (s) $\mathrm{cm}^{-1}$; MS (EI, $\left.70 \mathrm{eV}\right) \mathrm{m} / \mathrm{z}$ (rel int) $286.2\left(10,[\mathrm{M}+\mathrm{H}]^{+}\right), 230.1$ (28), 185.1 (37), 83.0 (64), 57.2 (100); HRMS (ESI, positive ion) calcd for $\mathrm{C}_{14} \mathrm{H}_{23} \mathrm{NO}_{5} \mathrm{Na}$ [M+Na $]^{+}$308.1474; found 308.1464 .
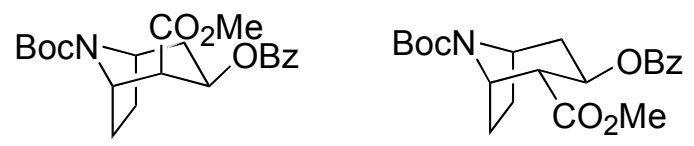

$(1 S, 2 S, 3 R, 5 R)$-3-Benzoyloxy-8-aza-bicyclo[3.2.1]octane-2,8-dicarboxylic acid 8-tertbutyl ester 2-methyl ester (15ax) and (1S,2R,3R,5R)-3-Benzoyloxy-8-azabicyclo[3.2.1]octane-2,8-dicarboxylic acid 8-tert-butyl ester 2-methyl ester (15eq): A solution of $\beta$-Hydroxy esters 14ax/eq (185 mg, $0.65 \mathrm{mmol}$ ), benzoic anhydride (294 mg, $1.3 \mathrm{mmol}$ ), and 4-dimethylaminopyridine (175 mg, $1.4 \mathrm{mmol})$ in methylene chloride (2.6 $\mathrm{mL}$ ) was stirred at room temperature for $12 \mathrm{~h}$. The mixture was diluted with ether and washed with water $(2 \mathrm{x})$, saturated aqueous $\mathrm{NaHCO}_{3}(1 \mathrm{x})$, and water $(1 \mathrm{x})$. The organic phase was dried $\left(\mathrm{MgSO}_{4}\right)$ and concentrated to afford an oil. Crude ${ }^{1} \mathrm{H}$ NMR showed a 1:1 mixture of $\mathrm{C}(2)$ epimers. Chromatography (10\% EtOAc/Hexanes) yielded $152 \mathrm{mg}$ 
(60\%) of 15ax/eq as an oil. The C(2) epimers were separated by HPLC (Dynamax Microsorb $\mathrm{C}_{60} 205 \times 21.4 \mathrm{~mm}$ column, $15 \% \mathrm{EtOAc} /$ Hexanes, $10 \mathrm{~mL} / \mathrm{min}$ at $254 \mathrm{~nm}$ ) to give 15eq $\left(t_{\mathrm{R}}=27.8 \mathrm{~min}\right)$ and $15 \mathbf{a x}\left(t_{\mathrm{R}}=38.7 \mathrm{~min}\right)$. Data for 15eq: $\mathrm{R}_{f}=0.63(30 \%$ EtOAc/Hexanes); $[\alpha]^{23}{ }_{\mathrm{D}}=+1.5^{\circ} ;{ }^{1} \mathrm{H}$ NMR $\left(500 \mathrm{MHz}, \mathrm{CDCl}_{3}\right) \delta 7.97(\mathrm{dd}, 2 \mathrm{H}, J=8.0$, $1.5 \mathrm{~Hz}), 7.54(\mathrm{tt}, 1 \mathrm{H}, J=7.0,1.5 \mathrm{~Hz}), 7.41(\mathrm{t}, 2 \mathrm{H}, J=8.0 \mathrm{~Hz}), 5.69(\mathrm{ddd}, 1 \mathrm{H}, J=10.7$, 10.7, $6.5 \mathrm{~Hz}), 4.51-4.25(\mathrm{~m}, 2 \mathrm{H}), 3.65$ (s, $3 \mathrm{H}), 3.08-2.90$ (m, $1 \mathrm{H}), 2.25(\mathrm{~s}, 1 \mathrm{H}), 2.08-$ $1.88(\mathrm{~m}, 4 \mathrm{H}), 1.85-1.81(\mathrm{~m}, 1 \mathrm{H}), 1.51(\mathrm{~s}, 9 \mathrm{H}) ;{ }^{13} \mathrm{C} \mathrm{NMR}\left(125 \mathrm{MHz}, \mathrm{CDCl}_{3}\right) \delta 171.2$, $165.5,153.0,133.0,130.1,129.6,128.3,80.1,67.8,55.2,54.8,53.3,52.7,52.0,51.0$, 49.9, 36.2, 35.5, 28.4, 25.6, 24.8; IR (neat) 1740 (s), 1716 (s), 1693 (s) $\mathrm{cm}^{-1}$; MS (EI, 70 eV) $m / z$ (rel int) $389.3\left(2,\left[\mathrm{M}^{+}\right]\right), 289.2$ (31), 168.1 (100), 108.1 (21), 57.2 (27); HRMS (EI, $70 \mathrm{eV}$ ) calcd for $\mathrm{C}_{21} \mathrm{H}_{27} \mathrm{NO}_{6}\left[\mathrm{M}^{+}\right]$389.1838; found 389.1831. Data for 15ax: $\mathrm{R}_{f}=$ $0.57\left(30 \%\right.$ EtOAc/Hexanes); $[\alpha]^{23}{ }_{\mathrm{D}}=+4.3^{\circ} ;{ }^{1} \mathrm{H} \mathrm{NMR}\left(500 \mathrm{MHz}, \mathrm{CDCl}_{3}\right) \delta 7.97(\mathrm{t}, 2 \mathrm{H}, J$ $=6.5 \mathrm{~Hz}), 7.54(\mathrm{t}, 1 \mathrm{H}, J=7.5 \mathrm{~Hz}), 7.40(\mathrm{t}, 2 \mathrm{H}, J=7.5 \mathrm{~Hz}), 5.47(\mathrm{dt}, 1 \mathrm{H}, J=11.7,6.0$ Hz), $4.68(\mathrm{~d}, 0.5 \mathrm{H}, J=6.5 \mathrm{~Hz}), 4.55(\mathrm{~s}, 1 \mathrm{H}), 4.36(\mathrm{~s}, 0.5 \mathrm{H}), 3.70(\mathrm{~s}, 1.5 \mathrm{H}), 3.68$ (s, 1.5 H), 3.07 (s, $1 \mathrm{H}), 2.57(\mathrm{td}, 1 \mathrm{H}, J=11.7,3.0 \mathrm{~Hz}), 2.14-2.08(\mathrm{~m}, 1 \mathrm{H}), 2.06-2.00(\mathrm{~m}, 1 \mathrm{H})$, 1.95-1.89 (m, $1 \mathrm{H}), 1.85-1.77(\mathrm{~m}, 2 \mathrm{H}), 1.47(\mathrm{~s}, 4.5 \mathrm{H}), 1.45(\mathrm{~s}, 4.5 \mathrm{H}) ;{ }^{13} \mathrm{C}$ NMR (125 $\left.\mathrm{MHz}, \mathrm{CDCl}_{3}\right) \delta 170.2,170.0,165.8,152.5,152.0,133.1,129.9,129.6,128.3,79.6,79.5$, $66.7,60.4,54.6,54.5,52.7,51.9,51.63,51.6,49.1,48.9,33.5,33.4,28.9,28.4,28.1$, 27.9, 27.2; IR (neat) 1740 (s), 1716 (s), 1693 (s) $\mathrm{cm}^{-1}$; MS (EI, $\left.70 \mathrm{eV}\right) \mathrm{m} / z$ (rel int) 389.3 (2, $\left.\left[\mathrm{M}^{+}\right]\right), 289.2$ (31), 168.1 (100), 108.1 (21), 57.2 (27); HRMS (EI, $70 \mathrm{eV}$ ) calcd for $\mathrm{C}_{21} \mathrm{H}_{27} \mathrm{NO}_{6}\left[\mathrm{M}^{+}\right]$389.1838; found 389.1831. 


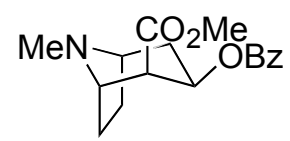

\section{M e t h y l $\quad(1 S, 2 S, 3 R, 5 R)-3$-(benzyloxy)-8-methyl-8-azabicyclo[3.2.1]octane-2-} carboxylate $((+)-1)$ : Trifluoroacetic acid $(0.21 \mathrm{~mL}, 310 \mathrm{mg}, 2.8 \mathrm{mmol})$ was added dropwise to a stirred solution of carbamate $15 \mathbf{a x}(31 \mathrm{mg}, 0.080 \mathrm{mmol})$ in methylene chloride $(0.95 \mathrm{~mL})$. Stirring was continued for $1 \mathrm{~h}$ at room temperature afterwhich the solvent was removed under reduced pressure. The crude residue was then partitioned between methylene chloride and saturated aqueous sodium bicarbonate. The aqueous layer was further extracted with methylene chloride (5x). The combined organic phases were dried $\left(\mathrm{Na}_{2} \mathrm{SO}_{4}\right)$, filtered and concentrated to give an oil. The crude oil was then taken up in acetonitrile $(1.9 \mathrm{~mL})$ and a $37 \%$ aqueous formaldehyde solution ( $35 \mu \mathrm{L}, 0.48$ mmol) was added followed by sodium cyanoborohydride $(10 \mathrm{mg}, 0.16 \mathrm{mmol})$. The reaction mixture was stirred for $1 \mathrm{~h}$ at room temperature. The mixture was then acidified to $\mathrm{pH} 6$ was glacial acetic acid. After stirring for an additional $0.5 \mathrm{~h}$ the solution was basidified to $\mathrm{pH}$ 8-9 with aqueous ammonium hydroxide. The mixture was partitioned between methylene chloride and saturated aqueous sodium bicarbonate. The aqueous layer was extracted with methylene chloride $(4 \mathrm{x})$. The combined organic phases were dried $\left(\mathrm{Na}_{2} \mathrm{SO}_{4}\right)$, filtered and concentrated. Chromatography (0-1\% methanol/methylene chloride, Neutral Alumina Brockman I) gave $17.9 \mathrm{mg}$ (74\%) of (+)-1 as a white solid. Data for (+)-1: $\mathrm{R}_{f}=0.57\left(5 \%\right.$ methanol/methylene chloride); m.p. $=92-94{ }^{\circ} \mathrm{C}$ (Lit. m.p. $=98{ }^{\circ} \mathrm{C}$ ); $[\alpha]_{\mathrm{D}}^{23}=+14.5^{\circ}$; er 93:7 (chiral HPLC, Chiralpak OD column, 10\% IPrOH/hexanes, $1 \mathrm{~mL} / \mathrm{min}$ at $\left.254 \mathrm{~nm}, t_{\mathrm{R}} 8.10 \mathrm{~min}\right) ;{ }^{1} \mathrm{H}$ NMR $\left(500 \mathrm{MHz}, \mathrm{CDCl}_{3}\right) \delta 8.02$ (dd, $2 \mathrm{H}, J=8.0,1.5 \mathrm{~Hz}), 7.53(\mathrm{tt}, 1 \mathrm{H}, J=7.5,1.5 \mathrm{~Hz}), 7.41$ (t, $2 \mathrm{H}, J=7.5 \mathrm{~Hz}), 5.24$ (dt, 
$1 \mathrm{H}, J=11.5,6.0 \mathrm{~Hz}), 3.71(\mathrm{~s}, 3 \mathrm{H}), 3.57-3.56(\mathrm{~m}, 1 \mathrm{H}), 3.30(\mathrm{~s}, 1 \mathrm{H}), 3.00(\mathrm{dd}, 1 \mathrm{H}, J=$ 8.5, $5.0 \mathrm{~Hz}), 2.43(\mathrm{t}, 1 \mathrm{H}, J=9.5 \mathrm{~Hz}), 2.23(\mathrm{~s}, 3 \mathrm{H}), 2.21-2.08$ (m, $2 \mathrm{H}), 1.89-1.85$ (m, 1 $\mathrm{H}), 1.73-1.71(\mathrm{~m}, 2 \mathrm{H}) ;{ }^{13} \mathrm{C} \mathrm{NMR}\left(125 \mathrm{MHz}, \mathrm{CDCl}_{3}\right) \delta 170.7,166.2,132.9,130.3,129.7$, 128.3, 66.9, 64.8, 61.6, 51.4, 50.2, 41.1, 35.5, 25.4, 25.2; IR ( $\left.\mathrm{CHCl}_{3}\right) 1746(\mathrm{~s}), 1713(\mathrm{~s})$, $1273(\mathrm{~s}) \mathrm{cm}^{-1}$; MS (EI, $\left.70 \mathrm{eV}\right) \mathrm{m} / z$ (rel int) $303.2\left(23,\left[\mathrm{M}^{+}\right]\right), 272.1$ (7), 182.1 (100), 82.0 (94); HRMS (EI, $70 \mathrm{eV}$ ) calcd for $\mathrm{C}_{17} \mathrm{H}_{21} \mathrm{NO}_{4}\left[\mathrm{M}^{+}\right]$303.1471; found 303.1481 . 